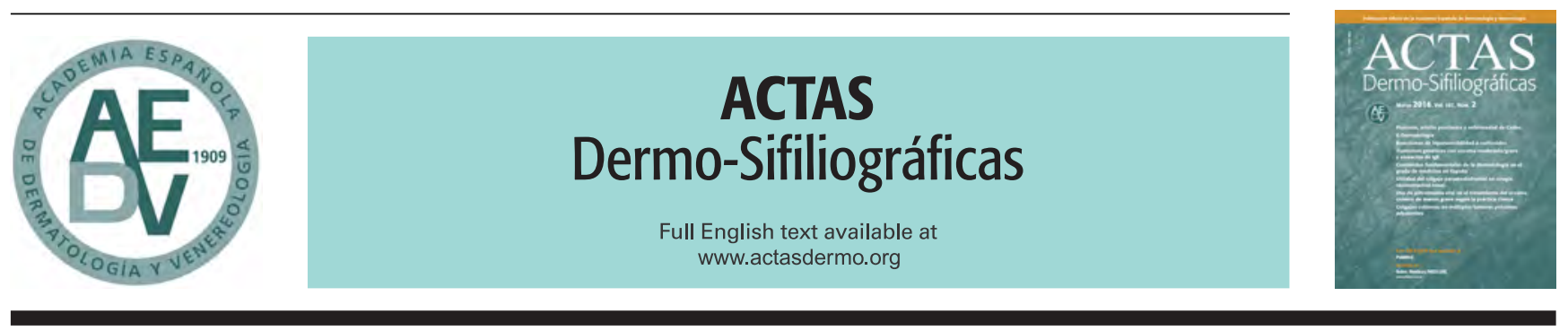

\title{
Desarrollo y validación preliminar del instrumento HSQoL-24 para evaluar calidad de vida en pacientes con hidradenitis supurativa
}

\author{
S.E. Marrón ${ }^{\mathrm{a}, \mathrm{h}, \mathrm{i}, *}$, M. Gómez-Barrera $^{\mathrm{b}, \mathrm{c}}$, L. Tomás-Aragonés ${ }^{\mathrm{g}, \mathrm{h}, \mathrm{i}}$, R.M. Díaz Díaz $^{\mathrm{d}, \mathrm{i}}$, \\ E. Vilarrasa Rull ${ }^{e}$, M.B. Madrid Álvarez ${ }^{\text {f,i }}$ y L. Puig ${ }^{\mathrm{e}}$
}

\footnotetext{
a Servicio de Dermatología, Hospital Royo Villanova, Zaragoza, España

b Universidad San Jorge, Villanueva de Gallego, Zaragoza, España

c Pharmacoeconomics \& Outcomes Research Iberia, Pozuelo de Alarcón, Madrid, España

d Servicio de Dermatología, Hospital Infanta Sofía, San Sebastián de los Reyes, Madrid, España

e Servicio de Dermatología, Hospital de la Santa Creu i Sant Pau, Barcelona, España

f Servicio de Dermatología, Hospital Dr. Negrín, Las Palmas de Gran Canaria, España

s Departamento de Psicología, Universidad de Zaragoza, Zaragoza, España

h Grupo Aragonés de Investigación en Psicodermatología (GAl+PD), Zaragoza, España

i Grupo Español de Investigación en Dermatología y Psiquiatría (GEDEPSI) de la Academia Española de Dermatología y Venereología (AEDV), Madrid, España
}

Recibido el 11 de noviembre de 2018; aceptado el 7 de febrero de 2019

\section{PALABRAS CLAVE \\ Hidradenitis supurativa; Calidad de vida; Resultados informados por los pacientes; Diseño de cuestionario; Validación psicométrica}

\begin{abstract}
Resumen
Introducción y objetivos: La hidradenitis supurativa (HS) es una enfermedad inflamatoria crónica de la piel que influencia negativamente la calidad de vida. En la actualidad no existen escalas en español que la evalúen. El objetivo del presente estudio fue desarrollar y validar un cuestionario específico para evaluar la calidad de vida en pacientes con HS.

Material y métodos: Se desarrolló un estudio multicéntrico en España entre 2016 y 2017 para elaborar un cuestionario. Para ello se consideró tanto el marco conceptual como el conocimiento de la situación del paciente mediante la revisión de la bibliografía, reuniones de profesionales de diferentes áreas y entrevistas con pacientes. El cuestionario resultante se pasó a un grupo de 30 pacientes con $30 \pm 10$ días de intervalo entre uno y otro.

Resultados: El análisis de fiabilidad muestra una buena consistencia interna y reproductibilidad con puntuación alfa de Cronbach de 0,920 (test) y 0,917 (retest) y coeficiente de correlación intraclase con DLQI y Skindex-29 de 0,698 IC 95\% (0,456-0,844) y 0,900 IC 95\% (0,801-0,951) respectivamente. Se establecieron puntos de corte para su uso y se comprobó que el instrumento es sensible al cambio.
\end{abstract}

\footnotetext{
* Autor para correspondencia.

Correo electrónico: semarron@aedv.es (S.E. Marrón).
} 


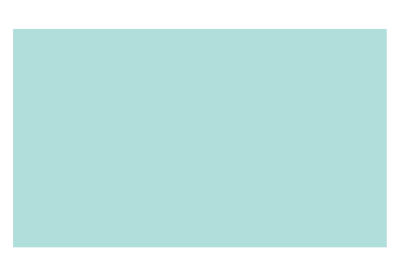

\section{KEYWORDS}

Hidradenitis

suppurativa;

Quality of life;

Patient-informed

results;

Questionnaire design;

Psychometric

validation
Conclusiones: El cuestionario HSQoL-24 es la primera prueba autoadministrada específica para evaluar la calidad de vida en HS en español. Sencillo de usar y puntuar por los profesionales. Este estudio demuestra que el instrumento es fiable, válido y sensible al cambio, pendiente de realizar estudio confirmatorio con una muestra mayor con 100 pacientes con HS. (c) 2019 AEDV. Publicado por Elsevier España, S.L.U. Todos los derechos reservados.

\section{Development and Preliminary Validation of the HSQoL-24 Tool to Assess Quality of Life in Patients With Hidradenitis Suppurativa}

\section{Abstract}

Introduction and objectives: Hidradenitis suppurativa (HS) is a chronic inflammatory disease of the skin with a negative impact on quality of life. Up to now, there are no disease specific instruments in Spanish to assess quality of life in HS. The objective of this study was to develop and validate a questionnaire to evaluate the quality of life in patients with HS.

Material and methods: A multicentre study was carried out in Spain between 2016 and 2017 to develop the questionnaire. Both the conceptual framework and understanding of the patient's situation were considered through a review of the literature, consensus of professionals from different related health areas, and in-depth interviews with patients. The resulting questionnaire was passed to a group of 30 patients with $30 \pm 10$ days of interval between both assessments.

Results: The reliability analysis shows a good internal consistency and reproducibility with Cronbach's alpha score of 0.920 (test) and 0.917 (retest) and intraclass correlation coefficient with DLQI and Skindex-29 of 0.698 IC 95\% (0.456-0.844) and 0.900 IC 95\% (0.801-0.951) respectively. Cut-off points were established for its use and the instrument was found to be sensitive to change.

Conclusions: The HSQoL-24 is the first disease-specific self-administered instrument to assess quality of life in patients with HS in Spanish. It is user friendly, and easy to score. This study shows that the instrument is reliable, valid and sensitive to change, pending confirmatory study with a larger sample of 100 patients with HS.

(c) 2019 AEDV. Published by Elsevier España, S.L.U. All rights reserved.

\section{Introducción}

La hidradenitis supurativa (HS) es una enfermedad cutánea inflamatoria crónica con afectación negativa a nivel físico y psicosocial ${ }^{1,2}$.

La calidad de vida se ve afectada por la $\mathrm{HS}^{3}$. Matusiak et al. ${ }^{4}$ realizan un análisis de las esferas de calidad de vida alteradas en la HS y describen una marcada alteración en las relaciones interpersonales del paciente con la pareja, con las relaciones sexuales, y con la familia, el desarrollo en algunos casos de ideas suicidas, y una frecuente pérdida de trabajo asociada a la enfermedad.

La aparición reciente de nuevos procedimientos «no invasivos» para el diagnóstico precoz de la enfermedad ${ }^{5}$, así como la de nuevas dianas terapéuticas van a revolucionar el abordaje de la $\mathrm{HS}^{6}$. En este nuevo escenario, se hace importante contar con un instrumento que mida la calidad de vida de los pacientes con HS, por ser una enfermedad que tiene su propia especificidad. Sin embargo, la revisión bibliográfica realizada mostró que no existe un instrumento en español para hacer esta medición.

El objetivo del presente estudio fue el desarrollo de un nuevo cuestionario psicométrico para evaluar la calidad de vida en pacientes con HS -el HSQoL-24- de acuerdo con los estándares actuales para su desarrollo.

\section{Material y métodos}

Se incluyeron en el estudio 30 pacientes adultos diagnosticados de HS. Todos firmaron documento de consentimiento informado. Para el presente estudio PI16/020 se obtuvo el Dictamen Favorable por parte del Comité Ético de Investigación Clínica de Aragón (CEICA) el 10 de febrero de 2016, así como los dictámenes favorables de los tres restantes hospitales. Los hospitales que reclutaron los pacientes fueron: Royo Villanova (Zaragoza), Infanta Sofía (Madrid), Santa Creu i Sant Pau (Barcelona) y Doctor Negrín (Las Palmas de Gran Canaria).

\section{Generación de ítems}

En primer lugar, se realizó una revisión bibliográfica en PubMed para identificar los determinantes psicosociales relevantes en la HS; estos sirvieron para identificar las áreas a explorar en las entrevistas. En segundo lugar, se realizaron entrevistas semiestructuradas a 12 pacientes ( 5 mujeres y 7 hombres con edades comprendidas entre 18 y 61 años) diagnosticados por un dermatólogo de HS. En tercer lugar, tras la transcripción y codificación de las entrevistas, se constituyó un panel de 20 expertos profesionales, con amplia experiencia clínica, para generar nuevos ítems. Con todo ello se 


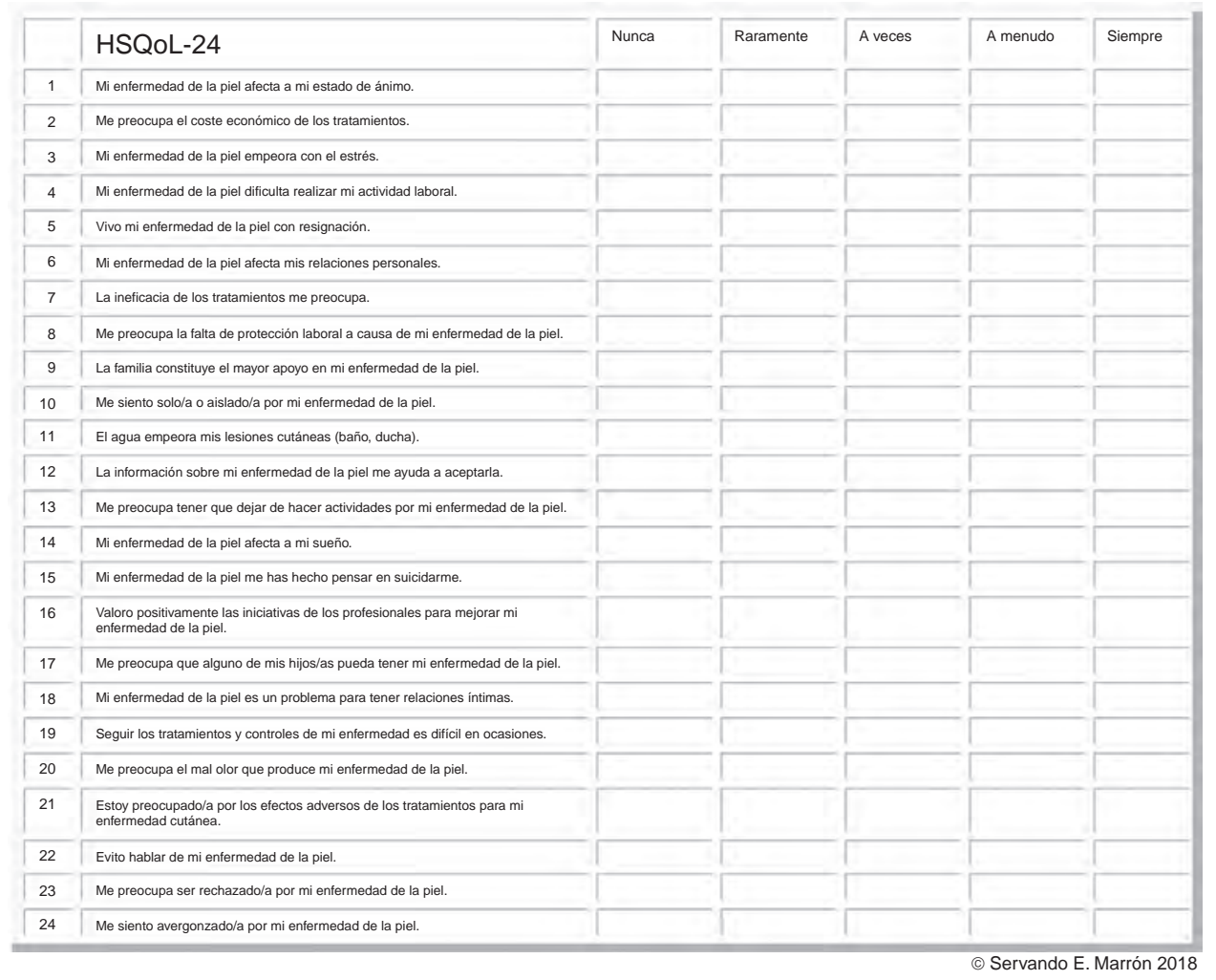

Figura 1 Cuestionario HSQoL-24.

elaboró un instrumento psicométrico de 24 ítems (HSQoL24). La temporalización de las preguntas es referida a las 4 semanas previas a la cumplimentación del cuestionario, ya que el curso evolutivo de la HS es relativamente estable a lo largo del tiempo en comparación con otras patologías cutáneas. Los ítems del cuestionario se agruparon en 6 dominios: psicosocial, económico, laboral, relacional, personal y clínico.

\section{Evaluación psicométrica}

Para la validación psicométrica de HSQoL-24 se utilizaron el Dermatology Life Quality Index (DLQI) ${ }^{7}$, cuestionario específico para la evaluación de la calidad de vida en enfermos dermatológicos, y el cuestionario Skindex-29 en su versión adaptada y validada al español por Jones-Caballero et al. ${ }^{8}$ que evalúa también calidad de vida dermatológica.

La fiabilidad se evaluó mediante análisis de consistencia interna con el estadístico alpha de Cronbach (es aceptable si el valor obtenido supera 0,7 ), y mediante análisis de reproductibilidad, evaluado con el coeficiente de correlación intraclase $(\mathrm{CCl})$, (considerándose adecuado si el valor obtenido supera 0,7$)$. La validez de contenido se evaluó por medio del juicio crítico de los 20 profesionales. La validez de constructo se realizó mediante análisis de correlación y regresión con los cuestionarios de calidad de vida DLQI y Skindex-29. Los valores de corte se calcularon mediante análisis de curvas de características operativas del receptor (COR) y comparación con las categorías de Skindex-29. La sensibilidad al cambio se analizó con las correlaciones entre las diferencias en las puntuaciones de los tres cuestionarios y con el tamaño del efecto.
Este cuestionario ha sido desarrollado por un grupo de expertos miembros del Grupo Aragonés de Investigación en Psicodermatología (GAl+PD) y del Grupo Español de Investigación en Dermatología y Psiquiatría (GEDEPSI) de la Academia Española de Dermatología y Venereología (AEDV). Se planteó una fase inicial de desarrollo en 30 pacientes, objeto del presente artículo y una posterior de confirmación en 100 pacientes. El cuestionario resultante, HsQoL-24 con 24 ítems y cinco posibles respuestas para cada uno (fig. 1), demostró ser factible y sencillo de realizar por los pacientes que invirtieron aproximadamente 10 min en sus respuestas.

\section{Resultados}

Se realizó la validación en una muestra de 30 pacientes, completando el HSQoL-24 en dos ocasiones con 30 \pm 10 días de diferencia entre ambas. De los 30 pacientes, 11 (36,7\%) fueron hombres, la media de edad ( \pm desviación estándar) fue de 36,8 $( \pm 11,2)$ años con una edad al inicio de 22,8 $( \pm 10,9)$ años y 13,6 $( \pm 11,3)$ años de duración de la enfermedad, 6 (20\%) Hurley III, 14 (46,7\%) Hurley II y $10(33,3 \%)$ Hurley I.

\section{Propiedades de HSQoL-24}

Los diferentes ítems se han agrupado en seis dominios: psicosocial: ítems $1,3,5,7,10,12,15,17,20,22,23$ y 24; económico: ítem 2; laboral: ítems 4 y 8 ; relacional: ítems 6 , 9, 13 y 18; personal; ítems 11 y 14; y clínico: ítems 16, 19 y 21. Para el cálculo de las puntuaciones, es necesario tener en cuenta que como norma general cada ítem se puntúa entre 0 y 4 , puntuando 0 la respuesta «nunca», 1 «raramente», 2 «a 
Tabla 1 Valor de las constantes de cada dominio

\begin{tabular}{ll}
\hline Dominio & Valores de la constante \\
\hline Global & 1,04166 \\
Psicosocial & 2,08333 \\
Económico & 25,0 \\
Laboral & 12,5 \\
Relacional & 6,25 \\
Personal & 12,5 \\
Clínico & 8,33 \\
\hline
\end{tabular}

Las puntuaciones de cada dominio y la puntuación global se dan referidas a 100 .

veces», 3 «a menudo» y 4 «siempre». Los ítems 9,12 y 16 se puntúan de forma inversa respecto a los demás. Finalmente, las puntuaciones de cada dominio y la puntuación general se deben ajustar a 100 , que se obtiene multiplicando el valor obtenido por una constante. Estas constantes se pueden ver en la tabla 1.

\section{Fiabilidad: consistencia interna y reproductibilidad}

\section{Consistencia interna}

La consistencia interna medida con el estadístico alpha de Cronbach presentó los valores de 0,920 en la validación inicial (o test) y 0,917 en la validación posterior (o retest).

\section{Reproductibilidad}

La reproductibilidad se midió con el $\mathrm{CCl}$ en una intervención test retest. Esta valoración se realizó para las puntuaciones totales y para los diferentes dominios. Los diferentes valores de los CCI de los cuestionarios DLQI y Skindex-29 suministrados a los pacientes fueron 0,698 IC 95\% (0,456 $0,844)$ y 0,900 IC 95\%: $(0,801-0,951)$ respectivamente. Los resultados se presentan en la tabla 2 .

\section{Análisis de la validez}

\section{Análisis de correlación y regresión}

La validez de constructo se realizó mediante análisis de correlación y regresión con los cuestionarios de CVRS DLQI y Skindex-29. Los valores de correlación del cuestionario
HSQoL-24 con el cuestionario DLQI y con el cuestionario Skindex-29 tanto en valoración pre- como en valoración posse presentan en la tabla 3. En esta tabla se puede constatar cómo los valores de correlación son mayores en la comparación con Skindex-29 y que se encuentran próximos a 0,9 $(0,863$ y 0,898 para test y retest respectivamente).

Se han realizado análisis de regresión del cuestionario HSQoL-24 con los cuestionarios DLQl y Skindex-29 en la valoración inicial y en la final. Los resultados de la regresión en los cuestionarios iniciales (test), se presentan en la tabla 4. Los valores de R2 presentados con Skindex-29 indicaban una adecuada bondad del ajuste del modelo $(0,7452$ y 0,7548 para el modelo test y retest respectivamente). De lo expuesto, se comprobó que el cuestionario DLQI permite predecir el 47-50\% del valor de la varianza en HSQoL-24 y el cuestionario Skindex-29 presenta un ajuste del modelo mayor, explicando el cuestionario Skindex-29 un $75-80 \%$ de la varianza en HSQoL-24.

\section{Puntos de corte}

Para delimitar los puntos de corte se compararon los rangos de valores de HSQoL-24 y Skindex-29 por ser con este último con quien presenta mejores valores de correlación y regresión. Los resultados del estudio descriptivo de los valores de HSQoL-24 en las diferentes categorías del cuestionario Skindex-29 se presentan en la tabla 5 . En esta tabla se puede ver que únicamente 8 y 10 pacientes no presentaron afectación en su calidad de vida y 14 y 13 pacientes presentaron afectación grave.

El análisis de los valores de las curvas COR determinan que los valores comprendidos entre 0-32 de HSQoL-24 corresponden a sujetos sin afectación en su CVRS con una sensibilidad de 0,909 y una especificidad de 0,5. El área bajo la curva es de 0,943 IC 95\% (0,865 -1,000). En cuanto a los pacientes con afectación grave, el punto de corte de la curva propuesto es de 42 con una sensibilidad de 0,933 y una especificidad de 0,867. El área bajo la curva es de 0,967 IC $95 \%(0,909-1,000)$. La separación en las categorías leve y moderada se propuso con el punto de corte 36 con sensibilidad 0,810 y especificidad 0,889 y área bajo la curva 0,910 IC $95 \%(0,807-1,000)$ (fig. 2$)$.

\section{Sensibilidad al cambio}

Para analizar la sensibilidad al cambio se calculó la variación de los tres cuestionarios y se realizó un análisis de correlación entre las mismas. Los resultados presentaron correlación estadísticamente significativa entre el cambio en HSQoL-24 y Skindex-29 (r de Pearson=0,504,

Tabla 2 Coeficiente de correlación intraclase

\begin{tabular}{lllll}
\hline Dominio & CCI (IC 95\%) & Media \pm DE, test & Media \pm DE, retest & p-valor, test retest $^{*}$ \\
\hline Global & $0,842(0,694-0,921)$ & $50,87 \pm 20,695$ & $47,60 \pm 19,866$ & 0,098 \\
Psicosocial & $0,872(0,750-0,937$ & $49,38 \pm 22,346$ & $45,76 \pm 21,949$ & 0,088 \\
Económico & $0,709(0,474-0,850)$ & $40,00 \pm 40,258$ & $40,00 \pm 40,258$ & 0,546 \\
Laboral & $0,682(0,431-0,835)$ & $51,67 \pm 34,072$ & $46,25 \pm 30,469$ & 0,259 \\
Relacional & $0,760(0,555-0,878)$ & $63,33 \pm 25,360$ & $59,79 \pm 23,309$ & 0,260 \\
Personal & $0,718(0,487-0,855)$ & $27,92 \pm 24,715$ & $29,17 \pm 24,197$ & 0,712 \\
Clínico & $0,702(0,462-0,846)$ & $58,61 \pm 19,013$ & $55,28 \pm 19,264$ & 0,227 \\
\hline
\end{tabular}

CCI: coeficiente de correlación intraclase; IC: intervalo de confianza; DE: desviación estándar.

p-valor de prueba t-Student. 
+Model

AD-2161; No. of Pages 7

Desarrollo y validación preliminar del instrumento HSQoL-24

Tabla 3 Análisis de correlaciones

\begin{tabular}{|c|c|c|c|c|}
\hline & \multicolumn{2}{|l|}{ Análisis test } & \multicolumn{2}{|l|}{ Análisis retest } \\
\hline & $\begin{array}{l}\text { Correlación con } \\
\text { DLQI (p-valor) }\end{array}$ & $\begin{array}{l}\text { Correlación con } \\
\text { Skindex-29 (p-valor) }\end{array}$ & $\begin{array}{l}\text { Correlación con } \\
\text { DLQI (p-valor) }\end{array}$ & $\begin{array}{l}\text { Correlación con } \\
\text { Skindex-29 (p-valor) }\end{array}$ \\
\hline Dominio global & $0,708(<0,001)$ & $0,863(<0,001)$ & $0,691(<0,001)$ & $0,898(<0,001)$ \\
\hline
\end{tabular}

DLQI: Dermatology Life Quality Index.

Tabla 4 Modelos de regresión

\begin{tabular}{|c|c|c|}
\hline Comparación & Modelo test (R2) & Modelo retest (R2) \\
\hline HSQoL-24 y DLQI & HSQoL=0,5732×DLQI+34,372 $(0,5018)$ & HSQoL=0,615×DLQI+32,604 $(0,4745)$ \\
\hline HSQoL-24 y Skindex-29 & HSQoL $=0,6907 \times$ Skindex $+21,057(0,7452)$ & HSQoL $=0,7548 \times$ Skindex $+18,418(0,8057)$ \\
\hline
\end{tabular}

R2: coeficiente de determinación múltiple.

Tabla 5 Valores de HSQoL-24 en categorías de Skindex-29

\begin{tabular}{|c|c|c|c|c|c|c|}
\hline \multirow[t]{2}{*}{ Categoría } & \multicolumn{3}{|c|}{ Test } & \multicolumn{3}{|c|}{ Retest } \\
\hline & Casos & Media $\pm \mathrm{DE}$ & Rango & Casos & Media $\pm \mathrm{DE}$ & Rango \\
\hline Sin afectación (0-24) & 8 & $29,82+6,51$ & $16-35$ & 10 & $27,90 \pm 10,35$ & $13-41$ \\
\hline Afectación leve (25-31) & 1 & 44,79 & 45 & 2 & $40,00 \pm 14,14$ & $30-50$ \\
\hline Afectación moderada (32-43) & 6 & $37,33 \pm 6,12$ & $31-47$ & 2 & $39,00 \pm 5,65$ & $35-43$ \\
\hline Afectación grave $(\geq 44)$ & 14 & $67,63 \pm 20,67$ & $35-83$ & 13 & $66,31 \pm 11,09$ & $45-83$ \\
\hline
\end{tabular}

DE: desviación estándar.
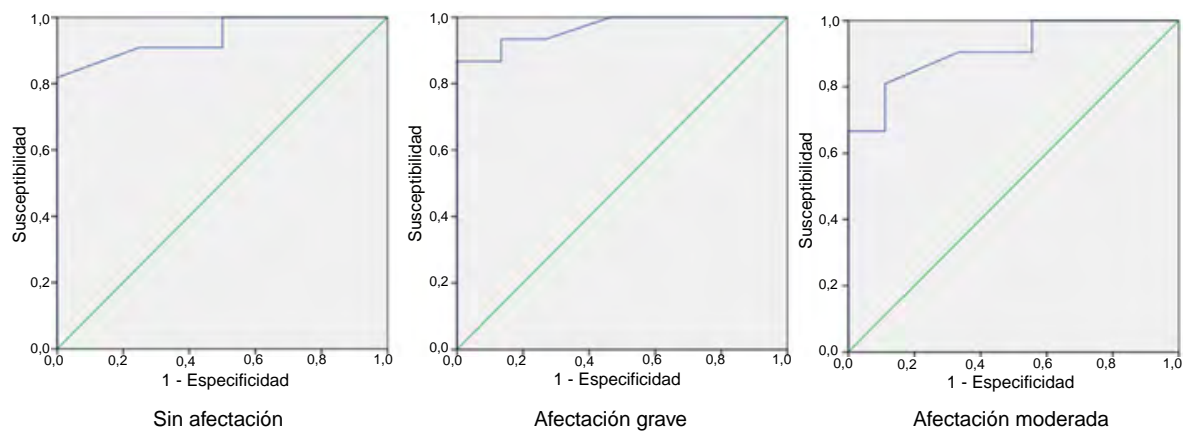

Figura 2 Curvas COR.

$\mathrm{p}=0,004)$ y no presentó correlación estadísticamente significativa, pero sí una tendencia con DLQI ( $r$ de Pearson=0,355, $\mathrm{p}=0,055)$. El tamaño del efecto de la variación de HSQoL24, calculado como diferencia de medias dividido por la desviación del momento inicial fue de 0,33 (50,87 $47,60 / 20,695)$, lo que indica una variación reducida en la CVRS. A parecidas conclusiones llegaba el cuestionario Skindex-29 con un valor de 0,17 (43,2-38,6/25,865); lo que indica en ambos casos una escasa variación en la CVRS en este tipo de pacientes en ambos cuestionarios.

Los valores en los tres cuestionarios demostraron diferencias en su puntuación global y en su puntuación final, los valores medios se presentan en la figura 3 y en ella

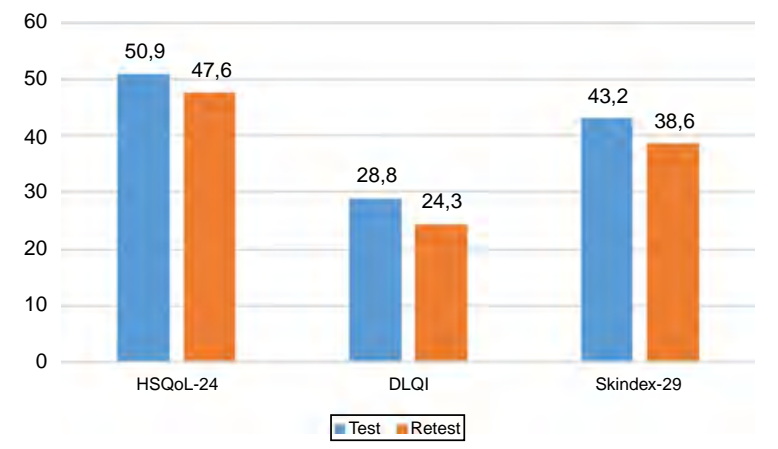

Figura 3 Comparativa de test retest de los tres cuestionarios. 
se ve cómo los valores se reducen en los tres valores medios.

\section{Discusión}

El presente artículo analiza las características del cuestionario HSQOL-24 como predictor de calidad de vida en pacientes con HS. En la elaboración previa del cuestionario se han considerado tanto el marco conceptual como el conocimiento de la situación del paciente mediante la revisión de la bibliografía, reuniones de profesionales de diferentes áreas y entrevistas con pacientes. Esta metodología de trabajo es consistente con la de otros autores y habitual en el desarrollo de un cuestionario de calidad de vida ${ }^{9,10}$.

La pertinencia de los estudios de calidad de vida en HS se ha puesto de manifiesto en varios estudios. Los pacientes más allá de sus manifestaciones clínicas ven afectada su calidad de vida y se deben analizar estas mermas ${ }^{11,12}$ así como los factores que influyen en las mismas ${ }^{13}$. Además de esta pertinencia existe la necesidad de generar cuestionarios aplicables a poblaciones específicas, en este caso la población española con HS, lo que justifica la necesidad de esta investigación en el momento actual de estudio de la enfermedad. Los cuestionarios disponibles actualmente sobre HS no son aplicables a población española hasta su traducción y validación y no se encontraban publicados en el momento del diseño y posterior recogida de datos del presente estudio. Ello explica que no se pudiera contemplar la traducción de los mismos y se optara por la realización de un cuestionario de novo $9,14,15$.

Las características psicométricas analizadas en el presente artículo han sido la fiabilidad, validez y capacidad pronóstica, medidas igualmente aceptadas en las diferentes enfermedades con afectación de la calidad de vida en España ${ }^{16}$.

La fiabilidad se midió como consistencia interna y/o reproductibilidad/repetibilidad. La consistencia interna se midió con el estadístico alpha de Cronbach ${ }^{17}$. Los resultados presentaron un valor superior a 0,9 lo que indica una consistencia interna excelente que se mantiene aun cuando se elimine alguna de las cuestiones. Parecidos valores fueron hallados por McLellan et al. ${ }^{16}$ que presentaron valores de alpha de 0,955 en la función física y 0,900 en el dominio de los síntomas en un cuestionario de calidad de vida en HS. Pinnard et al., notificaron asimismo valores elevados, $0,936^{15}$.

La reproductibilidad o repetibilidad se midió con el $\mathrm{CCl}$ en una intervención test-retest, asumiendo que resultan adecuados valores superiores a 0,7 . Esta valoración se realizó para las puntuaciones totales y para los diferentes dominios. Los valores del $\mathrm{CCl}$ superaron el valor de 0,7 en todos los dominios salvo en el laboral $(0,68)$. Estos valores son similares a los localizados en otros cuestionarios o métodos empleados en Dermatología, como pueda ser la determinación computadorizada de la medición Psoriasis Area and Severity Index (PASI) en psoriasis que alcanzaba valores de 0,86 IC $95 \%(0,80-0,90)^{18}$.

Los diferentes valores de los $\mathrm{CCl}$ de los cuestionarios DLQI y Skindex-29 presentaron amplios solapamientos con el IC 95\% de CCI de HSQoL-24, de lo que se dedujo que los valores de $\mathrm{CCl}$ de los tres cuestionarios presentaron valores comparables.

La validez de constructo se constató mediante análisis de correlación y regresión con los cuestionarios de CVRS DLQI y Skindex-29. Los valores elevados de los coeficientes de correlación y el adecuado ajuste de modelos de regresión indicaron adecuada validez, presentando una mayor corre- lación $(r>0,85)$ y mejor ajuste de regresión $\left(R^{2}>0,7\right)$. Al igual que otros autores en sus investigaciones ${ }^{15,16}$, HSQoL-24ha mostrado correlaciones adecuadas con estos cuestionarios lo que indica una validez adecuada.

Los valores de los puntos de corte presentaron una adecuada sensibilidad (entre 0,88 y 0,90 ) y una elevada área bajo la curva (superior a 0,9 ) en el análisis de curvas COR lo que indicaba una adecuada capacidad predictiva para detectar individuos con alteraciones en su CVRS. Estos valores fueron similares a los reportados por otros autores en otros cuestionarios en HS o Dermatología ${ }^{19,20}$. No obstante, no se pudieron calcular de forma adecuada los valores de especificidad. Ello puede ser atribuido al reducido tamaño de muestra, por lo que en la fase posterior de valoración se podrán analizar con mayor coherencia los valores de sensibilidad y especificidad del cuestionario HSQoL-24.

Además de las variaciones paralelas en las escalas de calidad de vida test y retest comentadas con anterioridad, la sensibilidad al cambio se analizó con el estudio de correlaciones entre las diferencias en las puntuaciones de los cuestionarios y con el tamaño del efecto. Los resultados presentaron correlación estadísticamente significativa entre el cambio en HSQoL-24 y Skindex-29, pero no con DLQI con un bajo valor de $p, p=0,055$. Los valores de correlación en torno a 0,5 fueron significativos y ligeramente inferiores a otros cuestionarios en Dermatología como el cuestionario Urticaria Activity Score que en su fase de validación alcanzaba valores de 0,6-0,7 en relación con otros cuestionarios ${ }^{21}$. No obstante, los resultados de HSQoL-24 se han considerado como indicadores de buena sensibilidad al cambio.

\section{Conclusiones}

Los resultados del análisis de las propiedades psicométricas de HSQoL-24, primer test específico autoadministrado para evaluar calidad de vida en pacientes con HS en español, demostraron unos valores adecuados de fiabilidad y validez lo que, a falta de los resultados de la validación en 100 pacientes, hacen de este cuestionario un elemento de amplia validez clínica en cuanto a resultados percibidos por los pacientes.

\section{Conflicto de intereses}

Los autores declaran no tener ningún conflicto de intereses.

\section{Bibliografía}

1. Fimmel $\mathrm{S}$, Zouboulis CC. Comorbidities of hidradenitis suppurativa (acné inversa). Dermatoendocrinol. 2010;2:9-16.

2. Kurzen H, Kurokawa I, Jemec GB, Emtestam L, Sellheyer K, Giamarellos-Bourboulis EJ, et al. What causes hidradenitis suppurativa? Exp Dermatol. 2008;17:455-6.

3. von der Werth JM, Jemec GB. Morbidity in patients with hidradenitis suppurativa. Br J Dermatol. 2001;144:809-13.

4. Matusiak L, Bieniek A, Szepietowski JC. Psychophysical aspects of hidradenitis suppurativa. Acta Derm Venereol. 2010;90:264-8

5. Martorell A, García-Martínez FJ, Jiménez-Gallo D, Pascual JC, Pereyra-Rodriguez J, Salgado L, et al. An update on hidradenitis suppurativa (Part I): Epidemiology, clinical aspects, and definition of disease severity. Actas Dermosifiliogr. 2015;106: 703-15. 
6. Martorell A, García FJ, Jiménez-Gallo D, Pascual JC, PereyraRodríguez J, Salgado L, et al. Update on hidradenitis suppurative (Part II): Treatment. Actas Dermosifiliogr. 2015;106: 716-24.

7. Finlay AY, Khan GK. Dermatology life quality index (DLQI) a simple practical measure for routine clinical use. Clin Exp Dermatol. 1994;19:210-6.

8. Jones-Caballero M, Peñas PF, García-Díez A, Chen MM, Badia X. The Spanish version of Skindex-29 An instrument for measuring quality of life in patients with cutaneous diseases. Med Clin (Barc). 2002;118:5-9.

9. Sisic M, Kirby JS, Boyal S, Plant L, McLellan C, Tan J. Development of a quality-of-life measure for hidradenitis suppurativa. J Cutan Med Surg. 2017;21:152-5.

10. Jiménez Hernández D, Tornel Abellán M, González Ortiz JJ. Optimización de un cuestionario mediante un método Delphi y una prueba piloto. RevllSE. 2015;3:617-38.

11. Kouris A, Platsidaki E, Christodoulou C, Efstathoiu V, Dessinioti C, Tzanekatou V, et al. Quality of life and psychosocial implications in patients with hidradenitis suppurativa. Dermatology. 2016;232:687-91.

12. Pavon Blanco A, Turner MA, Petrof G, Weinman J. To what extent do disease severity and illness perceptions explain depression, anxiety and quality of life in hidradenitis suppurativa. Br J Dermatol. 2019;180:338-45.

13. Kjaersgaard Andersen R, Theut Riis P, Jemec GBE. Factors predicting the self-evaluated health of hidradenitis suppurativa patients recruited from an outpatient clinic. J Eur Acad Dermatol Venereol. 2018;32:313-7.
14. McLellan C, Sisic M, Oon HH, Tan J. Preliminary Validation of the HS-QoL: A quality-of-life measure for hidradenitis suppurativa. J Cutan Med Surg. 2018;22:142-6.

15. Pinard J, Vieugels RA, Joyce C, Merola JF, Patel M. Hidradenitis suppurativa burden of disease tool: Pilot testing of a diseasespecific quality of life questionnaire. J Am Acad dermatol. 2018;78, 215-7.e2.

16. García de Yébenes Prous MJ, Rodríguez Salvanes F, Carmona Ortells L. Validación de cuestionarios. Reumatol Clin. 2009;5:171-7.

17. Crombach L. Coeffcient Alpha and the internal structure of test. Psychometrika. 1951;16:297-334.

18. Fink C, Alt C, Uhlmann L, Klose C, Enk A, Haenssle HA. Precision and reproducibility of automated computer-guided psoriasis area and severity index measurements in comparison to trained physicians. Br J Dermatol. 2019;180:390-6.

19. Lam C, Liu SW, Townsend HB, Femia AN, Qureshi AA, Vleugels RA. Development and pilot testing of the cutaneous lupus screening tool. JAMA Dermatol. 2016;152:60-6.

20. Esmann S, Dufour DN, Jemec GB. Questionnaire-based diagnosis of hidradenitis suppurativa: specificity, sensitivity and positive predictive value of specific diagnostic questions. $\mathrm{Br} \mathrm{J}$ Dermatol. 2010;163:102-16.

21. Hawro T, Ohanyan T, Schoepke N, Metz M, Peveling-Oberhag A, Staubach $\mathrm{P}$, et al. The urticaria activity score-validity, reliability, and responsiveness. J Allergy Clin Immunol Pract. 2018;6, -90 e 1185. 